\title{
Excimer laser atherectomy combined with drug-coated balloon angioplasty for the treatment of chronic obstructive femoropopliteal arterial disease
}

\author{
HUI LIU ${ }^{1,2}$, YAN GU $^{2}$, SEN YANG $^{2}, \mathrm{JU} \mathrm{HE}^{2}$ and FUXIAN ZHANG ${ }^{1}$ \\ ${ }^{1}$ Department of Vascular Surgery, Beijing Shijitan Hospital, Capital Medical University, Beijing 100038; \\ ${ }^{2}$ Department of Vascular Surgery, Tianjin First Central Hospital, Tianjin 300192, P.R. China
}

Received May 29, 2019; Accepted November 22, 2019

DOI: $10.3892 /$ etm.2019.8362

\begin{abstract}
The aim of the present retrospective study was to evaluate the feasibility, safety and the primary results following application of excimer laser atherectomy (ELA) combined with adjunctive drug-coated balloon angioplasty (DCBA) as the first-line endovascular treatment for patients with chronic obstructive femoropopliteal arterial disease. The baseline characteristics and angiographic variables of all patients who underwent ELA for stable chronic obstructive femoropopliteal arterial disease at Tianjin First Central Hospital (Tianjin, China) between May and December 2017 were collected. Information on clinical characteristics, including the 12-month primary patency rate, technical success rate, procedural success rate, bailout stenting rate, target lesion revascularization and major adverse events, was obtained following review of the patients' medical records. A descriptive analysis was performed on all variables. Kaplan-Meier curves were plotted for the primary patency rate. The present study

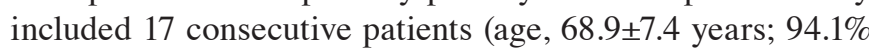
males) who were followed up for 12 months after the intervention. Adjunctive BA was performed in $100 \%$ of the cases. The occlusion length was $23.3 \pm 8.9 \mathrm{~cm}$ (range, $5.6-40.5 \mathrm{~cm}$ ). The technical success rate was $100 \%$ and the procedural success rate was $88.2 \%$. Bailout stenting was required in 5 of the 17 patients $(29.4 \%)$ and the 12-month primary patency rate was $82.4 \%$. The clinically driven target lesion revascularization rate was $5.9 \%$ at 12 months. An embolic protection device was used in $23.5 \%$ of the patients. The following adverse events were reported: Distal embolization requiring treatment, $5.9 \%$ (1 patient with embolic protection device); and flow-limiting dissection requiring treatment, 5.9\%. In the
\end{abstract}

Correspondence to: Dr Fuxian Zhang, Department of Vascular Surgery, Beijing Shijitan Hospital, Capital Medical University, 10 Tieyi Road, Yangfangdian, Haidian, Beijing 100038, P.R. China E-mail: tfhvascular@sina.com

Key words: excimer laser atherectomy, balloon angioplasty, drug-coated balloon, femoropopliteal artery, occlusion present study, there were no major adverse events (all-cause death, unplanned major amputation or target lesion revascularization) at 30 days after the intervention. Therefore, ELA combined with adjunctive DCBA for the treatment of chronic obstructive femoropopliteal arterial disease appears to be safe, practicable and associated with a high procedural success rate; furthermore, endoluminal-driven atherectomy may effectively reduce the requirement for stent placement in the lower limb arteries and is associated with long-term patency.

\section{Introduction}

Endovascular stent technology is a method commonly employed for treating peripheral arterial disease (PAD) and recent developments in this field have advanced conventional treatments, including percutaneous transluminal angioplasty (PTA), which is commonly used in patients with chronic lower limb PAD, e.g. elastic recoil, residual stenosis and flow-limiting dissection (1-6). However, secondary interventions may be required in several patients with PAD treated with stenting due to femoropopliteal in-stent restenosis, which is a regular defect associated with long and complex lesions $(7,8)$. It has been reported that femoropopliteal in-stent restenosis occurs during the first year of treatment in $18-40 \%$ of patients who have undergone stenting in the femoropopliteal segment $(9,10)$.

Aggressive debulking may be applied for intravascular plaque treatment, hyperproliferative endometrial tissue and clearance of thrombi. The debulking is performed using an excimer laser, an ultraviolet $(308 \mathrm{~nm})$ cold-tipped laserthat delivers ultra-short energy bursts (0.05 vs. $0.3-2.0 \mathrm{~mm} /$ pulse in the hot-tip laser). The photochemical, photothermal and photomechanical energy delivered by the excimer laser interacts with tissue, breaks down molecular bonds and creates vapor bubbles that generate kinetic energy. Long total superficial femoral artery (SFA) occlusions, as in the Peripheral Excimer Laser Angioplasty trial, as well as below-the-knee lesions in critical limb ischemia patients have been treated with excimer laser, providing improved results over those obtained with standard PTA alone, as well as excellent limb-salvage rates (11-13). The excimer laser probe is capable of 'step-by-step' debulking and hence, excimer laser atherectomy (ELA) outperforms other mechanical atherectomy treatments (14). 
With the advent of drug-coated balloon (DCB) technology, a potential novel treatment modality for femoropopliteal arterial disease has become available (with or without debulking). DCBs have been associated with inhibition of restenosis in several randomized studies on native SFA lesions with good results (15-17). DCBs alter the mitotic process of the smooth muscle cells and, in turn, confer improvements that may be sustained for at least 2 years. However, the effectiveness of DCB angioplasty (DCBA) has been reported to decline after the first year post-intervention $(18,19)$. The use of DCBA as an additional treatment following ELA has been proven to be superior to ELA treatment alone (20-22). In addition, the abovementioned studies have reported that DCBA treatment is critical for the success and sustained durability of ELA.

To date, little is known on ELA with adjunctive DCBA with regard to patency and target lesion revascularization rates associated with the treatment of stable chronic occlusive disease of the femoropopliteal artery. The aim of the present study was to assess the safety, feasibility and the primary results of ELA combined with adjunctive DCBA therapy in chronic obstructive femoropopliteal arterial disease.

\section{Materials and methods}

Study design and patient samples. The present study analyzed the clinical data of 17 consecutive patients with symptomatic chronic obstructive femoropopliteal arterial disease who were hospitalized at Tianjin First Central Hospital (Tianjin, China) between May and December 2017. The analysis included patients aged between 59 and 83 years who were diagnosed with either intermittent claudication or critical limb ischemia (Rutherford categories 3-5) (23). The patients' ankle-brachial index was $\leq 0.8$ in the target limb in all cases. All patients were treated with ELA (Turbo-Elite laser catheter, Spectranetics; Philips Medical Systems, Inc.), followed by angioplasty using DCBs (Orchid paclitaxel-coated balloon catheter; Acotec Scientific). In addition, Nitinol stents (Everflex; Medtronic Vascular, Inc.) were only implanted in cases with suboptimal results due to recoil or flow-limiting dissection.

The data of the present study included the baseline clinical characteristics, procedure, angiographic variables and outcomes that were extracted retrospectively, according to the electronic medical records and angiographic images at Tianjin First Central Hospital (Tianjin, China). Two physicians independently reviewed all angiographic images for lesion morphology and characteristics, according to the TransAtlantic Inter-Society Consensus (TASC) II classification system (6), and for distal run-off (24). Follow-up results were collected from all patients and their medical records were reviewed by a dedicated research coordinator.

In all patients, the onset of symptoms occurred $>6$ months prior to the primary crossing attempt. In patients with intermittent claudication, conservative medical treatment and exercise achieved no significant alleviation of the symptoms. The ankle-brachial index was determined prior to the intervention and in the follow-up examinations. All patients underwent a duplex ultrasound prior to the procedure; a focal increase in the peak systolic velocity of $\geq 140 \%$ (corresponding to a peak velocity ratio of $>2.4$ ) was considered indicative of a $>50 \%$ stenosis at that site (25). Pre-interventional diagnostics included CT angiography in all patients. The patients underwent follow-up evaluation at 12 months with duplex ultrasound and ankle-brachial index. Clinical examination was performed during the follow-up visits.

Definitions and outcome measures. The primary efficacy endpoint of the present study was the primary patency rate at 12 months. Secondary endpoints included the following: Technical success and acute procedural success rates, clinically driven target lesion revascularization during the 12-month follow-up, bailout stenting during the index procedure, ankle-brachial index and change in the Rutherford category score. Primary patency was defined as $\leq 50 \%$ diameter stenosis as assessed by duplex ultrasound in the absence of target lesion revascularization, amputation and/or surgical bypass. The patency threshold used for peak systolic velocity ratio was $\leq 2.4$. The technical success rate was defined as successful lesion crossing and treatment, with a final residual stenosis of $<30 \%$. Procedure success was defined as technical success with no major adverse events at the end of the procedure (26). Clinically driven target lesion revascularization was defined as a re-intervention performed for $>50 \%$ diameter stenosis or in the target lesion after documentation of recurrent clinical symptoms following the index procedure. The primary safety endpoints were evaluated as follows: Adverse events occurring during and after the intervention, prior to discharge, at the 30-day follow-up (including urgent target lesion thrombolysis/thrombectomy due to thrombosis/embolism, flow-limiting dissection, perforation, major bleeding and lower extremity bypass) and major adverse events (including all-cause death, unplanned major amputation and target lesion revascularization).

Endovascular procedure. All patients were treated using a standard technique and by the same operators, and were administered dual antiplatelet therapy [100 mg/day (d) aspirin and $75 \mathrm{mg} / \mathrm{d}$ clopidogrel] at the time of the procedure. Percutaneous access to the common femoral artery was obtained in all patients. The approach of contralateral crossover was preferred in the majority of cases. Unfractionated heparin was recommended during the endovascular procedure at sufficient doses (Initial dose, 100-150 U/kg; subsequent administrations, $50 \mathrm{U} / \mathrm{kg} / \mathrm{h}$ ) to maintain an activated clotting time of $\geq 250 \mathrm{sec}$. In all cases, a 7F or 8F Flexor Balkin Sheath or short sheath (Cook Medical) were employed to establish access. Stenosis/occlusion grade and lesion length were determined angiographically (Fig. 1A). The distal run-off status was also evaluated. The preferred technique used was endoluminal recanalization, which allowed for directly crossing the obstruction with the guidewire [0.035-inch Radiofocus angled soft hydrophilic (Terumo Medical); 0.018-inch V18 control wire (Boston Scientific)] and/or catheters [4F MPA catheter (Cordis); 2.6F CXI (Cook Medical)] avoiding the intentional subintimal approach. A total of seven cases suffered retrograde puncture of the normal artery at the distal end of the occlusion, or retrograde distal run-off vessels (SFA, posterior tibial artery, peroneal artery; Fig. 1B). The excimer laser (CVX-300 Excimer Laser System; Spectranetics; Philips Medical Systems, Inc.; wavelength, $308 \mathrm{~nm}$ ) was used and the Turbo-Elite laser catheter (2.0-2.5 mm, Spectranetics; Philips Medical Systems, Inc.) that was suitable for the vessel 

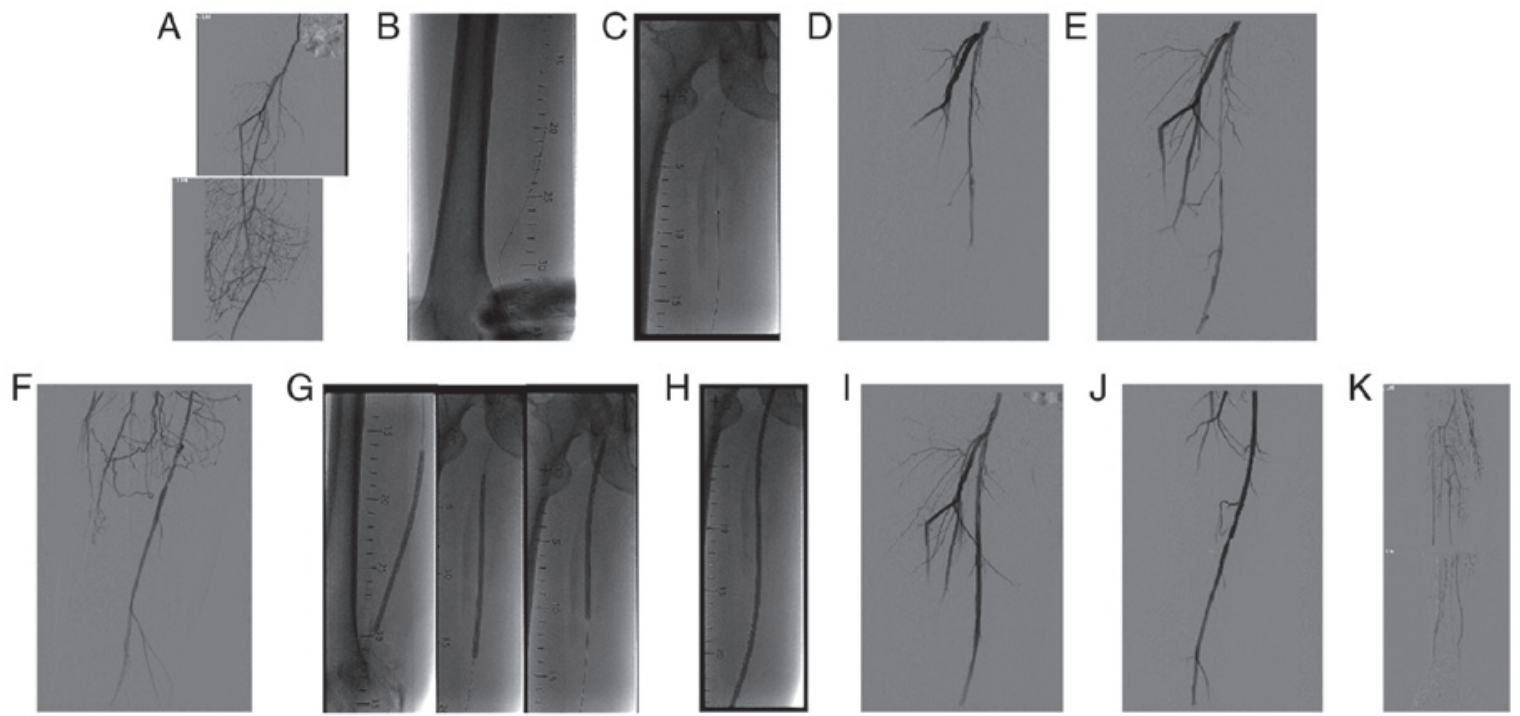

Figure 1. (A) Pre-treatment angiography, angiography image of a long total occlusion of the SFA. (B) Retrograde puncture access via popliteal artery P1 segment. (C) Turbo-Elite laser catheter during recanalization. (D) Post-ELA angiography, recanalized lumen of the proximal SFA after one passage of the laser catheter. (E) Post-ELA angiography, recanalized lumen of the mid-segment SFA after one passage of the laser catheter. (F) Post-ELA angiography, recanalized lumen of the distal SFA after one passage of the laser catheter. (G) Balloon angioplasty of the SFA. (H) Drug-coated balloon angioplasty of the SFA. (I) Final angiographic results of the proximal SFA. (J) Final angiographic results of the mid-segment and distal SFA. (K) Angiography for the artery below the knee. SFA, superficial femoral artery. Scale bar, $1 \mathrm{~cm}$.

diameter was inserted via a V18 control wire (Fig. 1C). The laser catheter was advanced $0.5-1 \mathrm{~mm} / \mathrm{sec}$. The angiographic results of ELA are depicted in Fig. 1D-F. Following ELA, PTA was routinely performed for $\geq 1 \mathrm{~min}$ with pressure-only balloons (Pacific/Admiral Xtreme/Amphirion Deep; Medtronic Vascular) matched to the reference vessel diameter (Fig. 1G). Subsequently, adjunctive DCBA was performed with a paclitaxel-coated $\left(3 \mu \mathrm{g} / \mathrm{mm}^{2}\right)$ balloon catheter (Orchid; Acotec Scientific) for $3 \mathrm{~min}$ (Fig. 1H). Bailout stenting was used for the treatment of flow-limiting dissection or suboptimal angiographic results with residual stenosis $(>30 \%)$. The use of a distal embolic protection device and the number of passes and energy delivered by ELA were at the operator's discretion. Completion angiography was then performed with distal run-off vessels to evaluate evidence of distal embolization. A successful procedure was assumed if a straight-line flow from the iliac arteries through $\geq 1$ tibial artery to the pedal arteries in the foot was achieved. The final angiographic results are depicted in Fig. 1I-K. A Perclose ProGlide Suture-Mediated Closure System (Abbott Vascular) was used for hemostasis of the common femoral artery access site and a bandage with mild compression was applied for $24 \mathrm{~h}$.

Patients were instructed to continue taking aspirin for $\geq 12$ months and clopidogrel for $\geq 6$ months following the procedure. Vessel calcification was graded angiographically using the following criteria as previously described (27): None/mild, no radio-opacities; moderate, radio-opacities appearing unilaterally on the arterial wall or for a length of $<1 \mathrm{~cm}$ prior to contrast injection or digital subtraction angiography; and severe, radio-opacities appearing bilaterally on the arterial wall and extending for a length of $>1 \mathrm{~cm}$ prior to contrast injection or digital subtraction angiography.

Statistical analysis. Continuous variables are expressed as the mean \pm standard deviation and categorical variables as $n(\%)$.
The primary patency rate was analyzed using Kaplan-Meier curves. $\mathrm{P}<0.05$ was considered to indicate a statistically significant difference. The pre-procedure ankle-brachial index was compared to the follow-up values using a paired t-test. Statistical analysis was performed using SPSS version 23.0 (IBM Corp.).

\section{Results}

Patient characteristics. The patients' demographic and clinical characteristics are summarized in Table I. The mean age of the patients was $68.9 \pm 7.4$ years and $94.1 \%$ of the patients were male. The patients had multiple comorbidities, with a high frequency of hypertension (76.5\%), hyperhomocysteinemia (58.8\%), cerebrovascular disease $(52.9 \%)$, smoking history $(76.5 \%)$ and diabetes $(52.9 \%)$. The baseline ankle-brachial index of the treated leg was $0.36 \pm 0.25$. The majority of the patients were severely symptomatic. In detail, a Rutherford category of 3 was determined in $52.9 \%$, a category of 4 in $17.7 \%$ and a category of 5 in $29.4 \%$ of the cases. The mean hospitalization time was $15.5 \pm 2.4$ days. The angiographic and procedural detailed characteristics are listed in Table II. The majority of the cases required a contralateral cross-over approach to cross the lesion $(88.2 \%)$ and 7 cases $(41.2 \%)$ required retrograde puncture of the normal artery at the distal end of the occlusion. Therefore, lesion crossing was achievable in all patients, including those with heavily calcified lesions, without the assistance of the step-by-step technique. The mean lesion length was $23.3 \pm 8.9 \mathrm{~cm}$ (range, $5.6-40.5 \mathrm{~cm}$ ) and $58.8 \%$ of the lesions were TASC II type D. All patients had chronic total occlusion in the femoropopliteal artery. Pre-interventional angiography revealed occlusion of the SFA in $41.2 \%$ and of the SFA + popliteal artery in $58.8 \%$ (including the P1-P3 segment), whereas certain patients also displayed iliac 
Table I. Demographic and clinical characteristics of the subjects $(\mathrm{n}=17)$.

\begin{tabular}{|c|c|}
\hline Variable & Value \\
\hline Age (years) & $68.9 \pm 7.4(59-83)$ \\
\hline Male & $16(94.1)$ \\
\hline \multicolumn{2}{|l|}{ Medical history } \\
\hline DM & $9(52.9)$ \\
\hline Hypertension & $13(76.5)$ \\
\hline Dyslipidemia & $4(23.5)$ \\
\hline CAD & $6(35.3)$ \\
\hline Prior PCI/bypass surgery & $2(11.8)$ \\
\hline CVD & $9(52.9)$ \\
\hline COPD & $1(5.9)$ \\
\hline Carotid stenosis & $6(35.3)$ \\
\hline Renal artery stenosis & $4(23.5)$ \\
\hline Hyperhomocysteinemia & $10(58.8)$ \\
\hline Homocysteine $(\mu \mathrm{mol} / \mathrm{ml})$ & $24.3 \pm 12.2(11.8-119.7)$ \\
\hline Current or former smoker & $13(76.5)$ \\
\hline History of other diseases ${ }^{a}$ & $4(23.5)$ \\
\hline Target lesion side ABI & $0.36 \pm 0.25(0-0.82)$ \\
\hline \multicolumn{2}{|c|}{ Pre-operative Rutherford category } \\
\hline 3 & $9(52.9)$ \\
\hline 4 & $3(17.7)$ \\
\hline 5 & $5(29.4)$ \\
\hline Hospital stay, days (range) & $15.5 \pm 2.4(9-28)$ \\
\hline Total cost, dollars (range) & $\begin{array}{l}18,689 \pm 3,347.3 \\
(11,576-25,941)\end{array}$ \\
\hline CTO & $17(100)$ \\
\hline \multicolumn{2}{|l|}{ Target lesion side } \\
\hline Right & $9(53.0)$ \\
\hline Left & $8(47.0)$ \\
\hline
\end{tabular}

${ }^{a}$ Nodular goiter, heart dysfunction, lumbar spondylosis. Continuous values are expressed as the means \pm standard deviation (range) and categorical data are presented as $\mathrm{n}(\%)$. DM, diabetes mellitus; CAD, coronary artery disease; PCI, percutaneous coronary intervention; CVD, cerebrovascular disease; COPD, chronic obstructive pulmonary disease; ABI, ankle-brachial index; CTO, chronic total occlusion.

artery lesions (17.7\%). The lesions were pre-treated with ELA and PTA in all cases. Turbo Elite catheters were used, mainly 2-mm (41.2\%), 2.3-mm (41.2\%) and 2.5-mm (17.6\%) in the femoral and popliteal segments. Proper lumen sizing was generally assured by angioplasty using long $(8-20-\mathrm{cm})$ balloons. The number of pressure-only balloons and DCBs was $2.8 \pm 1.2$ and $1.0 \pm 0.0 /$ patient, respectively. One TASC II type $\mathrm{D}$ patient experienced flow-limiting dissection. Bailout stenting was required in $5 / 17$ patients who were recanalized by ELA and adjunctive drug-coated BA due to flow-limiting dissection (5.9\% of the patients) and recoil (residual stenosis of $>30 \% ; 23.5 \%$ of the patients). The mean stent length was $182 \pm 50.4 \mathrm{~mm}$. The mean number of run-off vessels was $2.1 \pm 0.8$ /patient. A total of 6 patients had a 1 -vessel run-off, 4 had a 2 -vessel and 7 had a 3 -vessel run-off.
Table II. Detailed angiographic and procedural characteristics for the patients $(n=17)$.

\begin{tabular}{|c|c|}
\hline Variable & Value \\
\hline \multicolumn{2}{|l|}{ Access approach } \\
\hline Contralateral cross-over & $15 / 17(88.2)$ \\
\hline Antegrade & 2/17 (11.8) \\
\hline Adjunctive access & 7/17 (41.2) \\
\hline $\begin{array}{l}\text { Retrograde distal SFA/popliteal } \\
\text { artery P1 }\end{array}$ & $5 / 7(71.4)$ \\
\hline Retrograde PTA & $1 / 7(14.3)$ \\
\hline Retrograde peroneal artery & $1 / 7(14.3)$ \\
\hline \multicolumn{2}{|l|}{ TASC II } \\
\hline $\mathrm{B}$ & $5 / 17(29.4)$ \\
\hline $\mathrm{C}$ & 2/17 (11.8) \\
\hline $\mathrm{D}$ & $10 / 17(58.8)$ \\
\hline \multicolumn{2}{|l|}{ Lesion location } \\
\hline IA & 3/17 (17.7) \\
\hline Only SFA & $7 / 17(41.2)$ \\
\hline Only popliteal artery & $0 / 17(0)$ \\
\hline SFA and popliteal artery & $10 / 17(58.8)$ \\
\hline \multicolumn{2}{|l|}{ Calcification } \\
\hline None/mild & $10 / 17(58.8)$ \\
\hline Moderate & 3/17 (17.7) \\
\hline Severe & $4 / 17(23.5)$ \\
\hline FPA occlusion length $(\mathrm{cm})$ & $23.3 \pm 8.9(5.6-40.5)$ \\
\hline Below-knee runoff & $2.1 \pm 0.8$ \\
\hline 0 & $0 / 17(0)$ \\
\hline 1 & $6 / 17(35.3)$ \\
\hline 2 & 4/17 (23.5) \\
\hline 3 & $7 / 17(41.2)$ \\
\hline \multicolumn{2}{|l|}{ Laser parameters } \\
\hline Laser catheter size (mm) & $2.2 \pm 0.2$ \\
\hline 2.0 & $7 / 17$ (41.2) \\
\hline 2.3 & $7 / 17(41.2)$ \\
\hline 2.5 & 3/17 (17.6) \\
\hline Number of balloons/patient & $2.8 \pm 1.2$ \\
\hline Number of DCBs/patient & $1 \pm 0$ \\
\hline DCB diameter (mm) & $4.5 \pm 0.7$ \\
\hline DCB length (mm) & $210.9 \pm 90.0$ \\
\hline EPD & $4 / 17(23.5)$ \\
\hline Bailout stenting & $5 / 17(29.4)$ \\
\hline Stent length (mm) & $182 \pm 50.4$ \\
\hline \multicolumn{2}{|l|}{ Reason for bailout stenting } \\
\hline Recoil (residual stenosis $>30 \%$ ) & 4/17 (23.5) \\
\hline Flow-limiting dissection & $1 / 17(5.9)$ \\
\hline
\end{tabular}

Continuous values are expressed as the means \pm standard deviation (range) and categorical data are presented as n/total (\%). SFA, superficial femoral artery; PTA, posterior tibial artery; IA, Iliac artery; FPA, femoropopliteal artery; DCB, drug-coated balloon; EPD, embolic protection device; BTK, below the knee; ATA, anterior tibial artery; ELA, excimer laser atherectomy; TASC II, TransAtlantic Inter-Society Consensus II classification. 
Table III. Procedural outcomes.

\begin{tabular}{lc}
\hline Variable & Value \\
\hline Primary patency rate at 12 months & $14 / 17(82.4)$ \\
Technical success rate & $17 / 17(100)$ \\
Procedural success rate & $15 / 17(88.2)$ \\
Clinically-driven TLR at 12 months & $1 / 17(5.9)$ \\
Adverse events during hospitalization and & \\
at 30-day follow-up & \\
Embolism in all patients & $1 / 17(5.9)$ \\
Embolism in patients with EPD & $1 / 4(25)$ \\
Distal Embolization requiring treatment & $1 / 17(5.9)$ \\
Thrombosis & $0 / 17(0)$ \\
Flow-limiting dissection & $1 / 17(5.9)$ \\
Perforation & $0 / 17(0)$ \\
Major bleeding & $0 / 17(0)$ \\
MAEs & \\
All-cause death & \\
Unplanned minor/major amputation & $0 / 17(0)$ \\
TLR & $0 / 17(0)$ \\
\hline
\end{tabular}

The categorical data are presented as n/total (\%). EPD, embolic protection device; MAEs, major adverse events; TLR, target lesion revascularization.

Procedural outcomes. A summary of the procedural outcomes is provided in Table III. The primary patency rate was $82.4 \%$ at 12 months (Table III; Fig. 2), meeting the primary efficacy endpoint. Two patients with duplex ultrasound evidence of restenosis had an ankle-brachial index of $>0.7$ and were Rutherford category 1-2; therefore, repeat intervention was not performed. Technical and procedural success rates were 100 and $88.2 \%$, respectively. One TASC II type C patient experienced distal embolization intra-operatively, when balloon dilation of the target lesion after ELA resulted in emboli to the distal popliteal artery, which was successfully treated with rotational thrombectomy (Rotarex ${ }^{\circledR}$; Straub Medical). Another TASC II type D patient experienced flow-limiting dissection intra-operatively, which was successfully treated with bailout stenting. The rate of clinically driven target lesion revascularization at 12 months was $5.9 \%$ (1/17). One patient who reported rest pain was diagnosed with clinically significant acute re-occlusion with duplex ultrasound at 2 months post-intervention and underwent rotational thrombectomy due to acute thrombosis. In patients who underwent repeat intervention, there was restenosis at the edge of the stent. Ulcer healing were observed in all patients with immediate procedural success. None of the patients underwent amputation and no deaths were reported throughout the follow-up period. Table IV summarizes the changes in the ankle-brachial index immediately post-intervention and at 12 months compared with that pre-intervention. There was a significant improvement in the ankle-brachial index from $0.36 \pm 0.25$ prior to the intervention to a mean value of $0.94 \pm 0.13$ prior to discharge $(\mathrm{P}<0.001)$. In the follow-up period, no significant

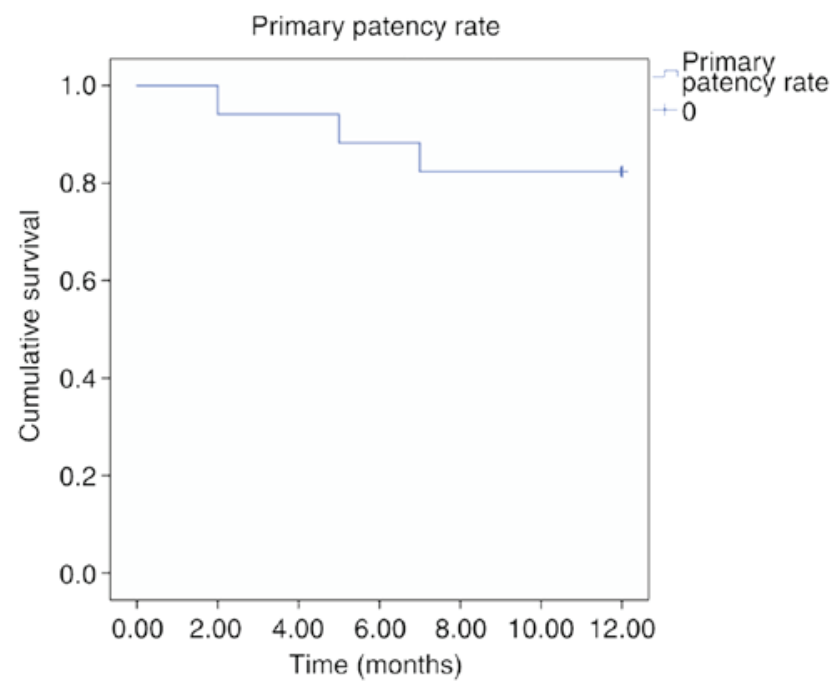

Figure 2. Primary patency rate displayed in a Kaplan-Meier curve.

decrease in the ankle-brachial index $(0.81 \pm 0.26)$ compared with the post-interventional control value $(0.94 \pm 0.13)$ was obtained $(\mathrm{P}>0.05)$. At 6 and 12 months, there were more patients with Rutherford categories of 0 and 1 compared with those at baseline (Fig. 3). Overall, the Rutherford category had improved at 12 months when compared with that at baseline. At baseline, 52.9, 17.7 and $29.4 \%$ of the patients were Rutherford category 3, 4 and 5, respectively, and at 12 months, 47.0, 29.4, 11.8 and $11.8 \%$ of the patients were Rutherford category 0,1 , 2 and 3 , respectively.

\section{Discussion}

SFA disease accounts for 40-50\% of patients with PAD (28). The first clinical manifestation is frequently the appearance of intermittent claudication. In patients with critical limb ischemia, more severe consequences may occur, which are associated with an increased risk of amputation and mortality (29). It is currently estimated that the incidence of PAD in Chinese patients aged $>60$ years is $20 \%$ (30). A study by Luo et al (31) demonstrated that the all-cause mortality rate for PAD was $22 \%$. Regarding treatment, bypass surgery is considered to be the gold standard for infra-inguinal chronic occlusions (32). However, patients, particularly diabetics, with critical limb ischemia are not considered as candidates for bypass surgery due to the multiple comorbidities and high surgical risk. Subsequently, endovascular therapy has been increasingly used due to its lower morbidity and mortality rates compared with those of bypass surgery. BA plus stenting is the most commonly used technique for revascularization in femoropopliteal arterial disease. However, revascularization of the femoropopliteal arteries with stable long-term results remains a challenge for endovascular specialists. While self-expanding Nitinol stents have demonstrated excellent immediate results, restenosis remains a limitation in the femoropopliteal segment, with restenosis rates of $20-37 \%$ at 12 months $(2,10,33)$ and $\leq 49 \%$ at 2 years (4). This makes in-stent occlusion another significant clinical stumbling block. Lesion crossing and recanalization of chronic total occlusions in the femoropopliteal arteries is often challenging. 
Table IV. Change in ABI in the patients ( $\mathrm{n}=17)$ across the study period.

P-value, vs.

Time-point

ABI F-value Pre-intervention

\begin{tabular}{lrrr}
\hline Pre-intervention & $0.36 \pm 0.25$ & & \\
Post-intervention & $0.94 \pm 0.13$ & 29.18 & $<0.001$ \\
Follow-up at 12 months & $0.81 \pm 0.26$ & 17.56 & $<0.001$ \\
\hline
\end{tabular}

ABI, Ankle-Brachial Index.

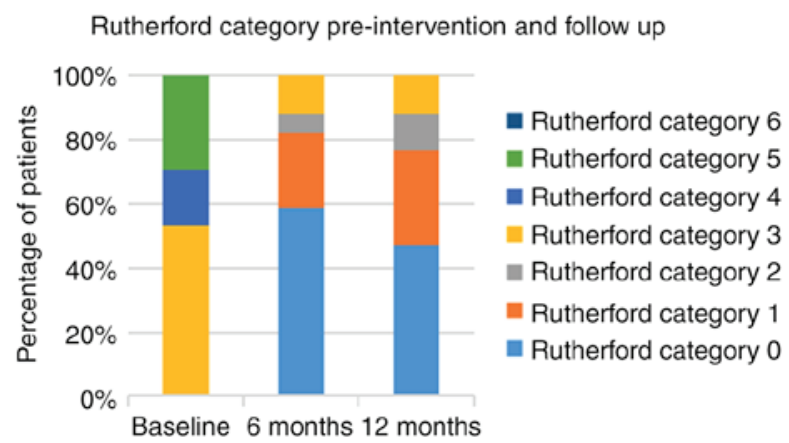

Figure 3. Rutherford category in patients with no target lesion revascularization at baseline, 6 months and 1 year after excimer laser treatment.

ELA involves employing photochemical, photomechanical and photothermal processes to debulk and ablate tissue (thrombi, atherosclerotic plaques) and has been proven to be a useful supplement to chronic obstructive arterial treatment $(11,34)$. The patency rates of successfully treated lesions (freedom from target lesion revascularization) have been reported to be $96.6 \%$ at 12 and $82.7 \%$ at 24 months (14). The same study also reported the expected limb-salvage rates at 12 and 24 months to be 100 and 94\%, respectively. Therefore, ELA appears to be an effective treatment for potential long occlusion and complex disease, since it provides a better angiographic result and lowers the requirement for stenting. High success rates of recanalization have been demonstrated in large earlier trials, even in long symptomatic SFA occlusions (35). In 2009, Dave et al (36) published a prospective multi-center study ( 17 hospitals in the US) evaluating the safety and efficacy of ELA in the treatment of primary femoropopliteal artery occlusive disease. Their study included 65 patients with a mean lesion length of $5.6 \mathrm{~cm}$, of which 13 cases were occluded; the mean lumen loss rate was reduced from 77 to $34.7 \%$, which was reduced to $21 \pm 14.5 \%$ after adjunctive therapy with BA $(\mathrm{n}=42,64.6 \%)$ or $\mathrm{BA}+$ stenting $(\mathrm{n}=15,23.3 \%)$, and the patency rate was 59 and $54 \%$ at 6 and 12 months, respectively. Target lesion revascularization was not required in 76.9\% of CliRpath Excimer Laser System to Enlarge Lumen Openings participants within the 1-year follow-up period. The safety and efficacy of this technique were validated in 65 patients without major adverse events or complications. Simultaneously, DCBs have achieved satisfactory results in the treatment of lower extremity occlusive primary lesions and in-stent restenosis $(15,16,19,37)$. LEVANT-2 is a large prospective, multi-center, single-blinded randomized controlled trial (RCT) comprising a total of 476 patients. Comparing the effects of DCB (Lutonix ${ }^{\mathrm{TM}}$; Bard) and PTA in the treatment of femoropopliteal artery occlusive disease, the results demonstrated that the patency rate of the DCB group was significantly higher compared with that of normal PTA at 1 year (65.2 vs. $52.6 \%$, respectively; $\mathrm{P}=0.015$ ) (38). IN.PACT SFA is also a prospective, multicenter RCT with a total of 331 patients enrolled. The patients were randomly assigned to the DCB (IN.PACT Admiral; Medtronic) and the normal PTA groups, and the results demonstrated that the target lesion revascularization of the DCB group was significantly lower compared with that in the normal PTA group (2.4 vs. $20.6 \%$, respectively; $\mathrm{P}=0.001)$. The 1 -year patency rate was $82.2 \%$ in the DCB and $52.5 \%$ in the normal PTA group, respectively $(\mathrm{P}=0.001)$ (39). In addition, ELA has been employed for the treatment of femoropopliteal in-stent restenosis (27). Specifically, Dippel et al (27) observed that ELA combined with PTA demonstrated superior procedural success compared with the PTA alone group (93.5 vs. 82.7\%; $\mathrm{P}<0.01$ ) and significantly fewer procedural complications. At 6 months, freedom from target lesion revascularization with ELA combined with PTA compared with PTA alone was observed in 73.5 vs. $51.8 \%$ of the patients, respectively $(\mathrm{P}<0.005)$, and the 30-day major adverse event rates were 5.8 vs. $20.5 \%(\mathrm{P}<0.001)$, respectively. The above-mentioned study concluded that ELA combined with PTA significantly lowered target lesion revascularization by $52 \%$ (hazard ratio $=0.48 ; 95 \% \mathrm{CI}, 0.31-0.74$ ).

The present study was a retrospective analysis aiming to evaluate the role of ELA combined with adjunctive DCBA in the treatment of femoropopliteal arterial disease. Previously published studies (the LACI phase 2 and LACI Belgium studies) have reported on the success of ELA-assisted angioplasty in the lower extremities $(12,13)$. The LACI phase 2 study evolved from a 25-patient phase I registry to enrollment of 145 patients with critical limb ischemia. Restoration of the straight-line flow to the foot was achieved in $89 \%$ of the patients, and adjunctive PTA and stenting were employed in 96 and $45 \%$ of cases, respectively, with a patency rate at 6 months of $83 \%$. The 6-month limb-salvage rate was $92.5 \%$, with a major amputation rate of $8 \%$. The LACI Belgium study included 48 patients with critical limb ischemia (13). In the majority of the patients, the straight-line flow to the foot was restored, and adjunctive PTA and stenting were used in 80 and $52.9 \%$ of cases, respectively. The 6-month patency rate was at $76 \%$ and the limb salvage rate at 6 months was $90.5 \%$, with a major amputation rate of $8 \%$.

Comparison of the results of the present study with those reported by the above-mentioned studies $(12,13)$ indicated similar lesion site observations and similar clinical characteristics of the patients. The LACI 2 and the LACI Belgium studies only included patients who were poor surgical candidates; however, in the present study, all patients with chronic total occlusion undergoing peripheral revascularization procedures were included, and ELA was employed in combination with DCBA in order to treat femoropopliteal arterial disease. This change in the patient criteria may explain the differences in the patency rates (82.4\% at 12 months) as well as bailout stenting rates (29.4\%) between the present study and the two LACI trials. Other possible explanations may be the reduced 
use of stents, smaller patient samples, the selection criteria and the single-center design of the present study. The ELA technique offers a theoretical advantage when compared with PTA, which is the reduced stretching of the vessel achieved by the ablation effect that allows for remodeling of the vascular lumen. Satisfactory long-term patency and limb-salvage rates are supported by the results of the present study and are in agreement with other published results on mechanical atherectomy (Rotarex or TurboHawk) (40). The in-stent restenosis incidence is more frequently encountered among diabetic patients and, during long-term endovascular intervention therapy, the high incidence of in-stent restenosis may lead to unproficient use of stents. Subsequently, ELA may offer better results than DCBA, as well as reduce the requirement for stenting.

An experience with 40 patients undergoing unsuccessful angioplasty of TASC C and D lesions in femoropopliteal arteries treated via ELA was reported by Wissgott et al (41). Their study reported a mean occlusion length of $17.5 \mathrm{~cm}$ (range, 12-25 cm). Recanalization using the step-by-step method of crossing was performed with an excimer laser catheter (catheter diameter range, 1.7-2.5 mm). Balloon dilation was performed in all cases after successful crossing and stent placement was required in $10 \%$ of the procedures. The initial technical success rate of $90 \%(36 / 40)$ resulted in a primary patency rate of $58.9 \%$ after 12 months. No serious complications attributable to the intervention were reported. The present study involved significantly longer occlusion lesions (23.3 vs. $17.5 \mathrm{~cm})$ and more TASC II D cases compared with those in the above-mentioned study. In addition, DCBs were used in the present study, which may explain for the higher primary patency and lower target lesion revascularization rates in the present study. Furthermore, all patients exhibited a marked improvement in their Rutherford category and their ankle-brachial index that persisted at the 12-month follow-up.

Lüdtke et al (42) reported that 15 patients $(15 / 67,22 \%)$ who underwent ELA-assisted PTA of the femoropopliteal arteries were treated via a retrograde transpopliteal approach. Technically successful recanalization was achieved in 14 of the 15 patients. There were no major adverse events regarding the laser atherectomy or popliteal access site. Compared with the results reported by Lüdtke et al (42), 7 patients of the present study required a retrograde recanalization access (including distal SFA/popliteal artery P1 segment, posterior tibial artery and peroneal artery). The technical success rate was $100 \%$. Retrograde access achieves a high recanalization rate in femoropopliteal arterial disease when conventional crossing attempts have failed. An advantage of the retrograde approach may be easier entry into the occlusion due to a softer distal cap. The popliteal approach was used more frequently compared with other access approaches. Sangiorgi et al (43) reported technical success rates of $\sim 96 \%$ in long TASC C and $\mathrm{D}$ lesions, and proposed the transpopliteal access as a safe and effective alternative when antegrade recanalization attempts have failed.

The present study takes into consideration the cost of ELA combined with DCB in the treatment of femoropopliteal arterial disease, and cost is one of the drawbacks of laser technology. It was estimated that the cost of ELA combined with DCB was higher compared with that of standard techniques
(PTA and stenting), with a mean cost of 18,689 dollars per procedure.

The limitations of the present study include its observational retrospective design, the small patient sample and the lack of a control group with the use of angioplasty combined with stenting for comparison. To date, no published randomized data are available to evaluate the efficacy of ELA combined with that of adjunctive DCBA in the treatment of chronic obstructive femoropopliteal arterial disease.

The results of the present study suggest that the ELA technique achieved a good technical success rate with a low complication rate in the treatment of TASC II B, C and D lesions. The use of ELA reduced the requirement for stenting in the lower limb arteries. The present study also demonstrated that the ELA approach may be valuable and effective even in long, complex occlusive lesions. Therefore, future large-scale randomized studies addressing complex cases of obstructive femoropopliteal arterial disease should be encouraged.

\section{Acknowledgements}

Not applicable.

\section{Funding}

This study was financially supported by the Science and TechnologyPlanProject of Tianjin (grant no. 18ZXDBSY00110) and the Tianjin Health and Family Planning Commission Science and Technology Fund Project (grant no. 2015KZ033).

\section{Availability of data and materials}

The datasets used and/or analyzed during the present study are available from the corresponding author on reasonable request.

\section{Authors' contributions}

Conception and design: HL and FZ. Analysis and interpretation: HL, FZ and JH. Data collection: HL, YG and SY. Writing of the manuscript: HL, YG, SY, JH and FZ. Critical revision of the manuscript: HL, JH and FZ. Final approval of the manuscript: HL, YG, SY, JH and FZ. Statistical analysis: HL. Overall responsibility: FZ.

\section{Ethics approval and consent to participate}

The study was performed in accordance with the ethical principles of the Declaration of Helsinki. The study protocol was reviewed and approved by the Tianjin First Central Hospital (Tianjin, China) institutional review board and all procedures were performed after obtaining informed consent from all patients.

\section{Patient consent for publication}

Not applicable.

\section{Competing interests}

The authors declare that they have no competing interests. 


\section{References}

1. Ferreira M,Lanziotti L, Monteiro M, Abuhadba G, Capotorto LF, Nolte L and Fearnot N: Superficial femoral artery recanalization with self-expanding nitinol stents: Long-term follow-up results. Eur J Vasc Endovasc Surg 34: 702-708, 2007.

2. Krankenberg H, Schlüter M, Steinkamp HJ, Bürgelin K, Scheinert D, Schulte KL, Minar E, Peeters P, Bosiers M, Tepe G, et al: Nitinol stent implantation versus percutaneous transluminal angioplasty in superficial femoral artery lesions up to $10 \mathrm{~cm}$ in length: The femoral artery stenting trial (FAST). Circulation 116: 285-292, 2007.

3. Zeller T, Tiefenbacher C, Steinkamp HJ, Langhoff R, Wittenberg G, Schlüter M, Buergelin K, Rastan A, Krumsdorf U, Sixt S, et al: Nitinol stent implantation in TASC A and B superficial femoral artery lesions: The femoral artery conformexx trial (FACT). J Endovasc Ther 15: 390-398, 2008.

4. Schillinger M, Sabeti S, Dick P, Amighi J, Mlekusch W, Schlager O, Loewe C, Cejna M, Lammer J and Minar E: Sustained benefit at 2 years of primary femoropopliteal stenting compared with balloon angioplasty with optional stenting. Circulation 115 : 2745-2749, 2007

5. Rocha-Singh KJ, Jaff MR, Crabtree TR, Bloch DA and Ansel G; VIVA Physicians, Inc: Performance goals and endpoint assessments for clinical trials of femoropopliteal bare nitinol stents in patients with symptomatic peripheral arterial disease. Catheter Cardiovasc Interv 69: 910-919, 2007.

6. Norgren L, Hiatt WR, Dormandy JA, Nehler MR, Harris KA, Fowkes FG; TASC II Working Group, Bell K, Caporusso J, Durand-Zaleski I, et al: Inter-society consensus for the management of peripheral arterial disease (TASCII). Eur J Vasc Endovasc Surg 33 (Suppl 1): S1-S75, 2007.

7. Gur I, Lee W, Akopian G, Rowe VL, Weaver FA and Katz SG: Clinical outcomes and implications of failed infrainguinal endovascular stents. J Vasc Surg 53: 658-666, 2011.

8. Armstrong EJ, Saeed H, Alvandi B, Singh S, Singh GD, Yeo KK, Anderson D, Westin GG, Dawson DL, Pevec WC and Laird JR: Nitinol self-expanding stents vs. balloon angioplasty for very long femoropopliteal lesions. J Endovasc Ther 21: 34-43, 2014

9. Schillinger M, Sabeti S, Loewe C, Dick P, Amighi J, Mlekusch W, Schlager O, Cejna M, Lammer J and Minar E: Balloon angioplasty versus implantation of nitinol stents in the superficial femoral artery. N Engl J Med 354: 1879-1888, 2006.

10. Laird JR, Katzen BT, Scheinert D, Lammer J, Carpenter J, Buchbinder M, Dave R, Ansel G, Lansky A, Cristea E, et al: Nitinol stent implantation versus balloon angioplasty for lesions in the superficial femoral artery and proximal popliteal artery: Twelve-month results from the RESILIENT randomized trial. Circ Cardiovasc Interv 3: 267-276, 2010.

11. Scheinert D, Laird JR Jr, Schröder M, Steinkamp H, Balzer JO and Biamino G: Excimer laser-assisted recanalization of long, chronic superficial femoral artery occlusions. J Endovasc Ther 8: $156-166,2001$

12. Laird JR, Zeller T, Gray BH, Scheinert D, Vranic M, Reiser C and Biamino G; LACI Investigators: Limb salvage following laser-assisted angioplasty for critical limb ischemia: Results of the LACI multicenter trial. J Endovasc Ther 13: 1-11, 2006.

13. Bosiers M, Peeters P, Elst FV, Vermassen F, Maleux G, Fourneau I and Massin H: Excimer laser assisted angioplasty for critical limb ischemia: Results of the LACI Belgium study. Eur J Vasc Endovasc Surg 29: 613-619, 2005.

14. Serino F, Cao Y, Renzi C, Mascellari L, Toscanella F, Raskovic D, Tempesta P, Bandiera G and Santini A: Excimer laser ablation in the treatment of total chronic obstructions in critical limb ischaemia in diabetic patients. Sustained efficacy of plaque recanalisation in mid-term results. Eur J Vasc Endovase Surg 39: 234-238, 2010

15. Werk M, Langner S, Reinkensmeier B, Boettcher HF, Tepe G, Dietz U, Hosten N, Hamm B, Speck U and Ricke J: Inhibition of restenosis in femoropopliteal arteries: Paclitaxel-coated versus uncoated balloon: Femoral paclitaxel randomized pilot trial. Circulation 118: 1358-1365, 2008.

16. Tepe G, Zeller T, Albrecht T, Heller S, Schwarzwälder U, Beregi JP, Claussen CD, Oldenburg A, Scheller B and Speck U: Local delivery of paclitaxel to inhibit restenosis during angioplasty of the leg. N Engl J Med 358: 689-699, 2008.

17. Ansel GM and Lumsden AB: Evolving modalities for femoropopliteal interventions. J Endovasc Ther 16 (Suppl 2): II82-II97, 2009.
18. Grotti S, Liistro F, Angioli P, Ducci K, Falsini G, Porto I, Ricci L, Ventoruzzo G, Turini F, Bellandi G and Bolognese L: Paclitaxel-eluting balloon vs standard angioplasty to reduce restenosis in diabetic patients with in-stent restenosis of the superficial femoral and proximal popliteal arteries: Three-year results of the DEBATE-ISR study. J Endovasc Ther 23: 52-57, 2016.

19. Virga V, Stabile E, Biamino G, Salemme L, Cioppa A, Giugliano G, Tesorio T, Cota L, Popusoi G, Pucciarelli A, et al: Drug-eluting balloons for the treatment of the superficial femoral artery in-stent restenosis: 2-year follow-up. JACC Cardiovasc Interv 7: 411-415, 2014

20. Kokkinidis DG, Hossain P, Jawaid O, Alvandi B, Foley TR, Singh GD, Waldo SW, Laird JR and Armstrong EJ: Laser atherectomy combined with drug-coated balloon angioplasty is associated with improved 1-year outcomes for treatment of femoropopliteal in-stent restenosis. J Endovasc Ther 25: 81-88, 2018.

21. Van den Berg JC, Pedrotti M, Canevascini R, Chimchila Chevili S, Giovannacci L and Rosso R: In-stent restenosis: Mid-term results of debulking using excimer laser and drug-eluting balloons: Sustained benefit? J Invasive Cardiol 26: 333-337, 2014.

22. Sixt S, Carpio Cancino OG, Treszl A, Beschorner U, Macharzina R, Rastan A, Krankenberg H, Neumann FJ and Zeller T: Drug-coated balloon angioplasty after directional atherectomy improves outcome in restenotic femoropopliteal arteries. J Vasc Surg 58: 682-686, 2013.

23. Rutherford RB, Baker JD, Ernst C, Johnston KW, Porter JM, Ahn S and Jones DN: Recommended standards for reports dealing with lower extremity ischemia: Revised version. J Vasc Surg 26: 517-538, 1997.

24. Popma JJ, Tiroch K, Almonacid A, Cohen S, Kandzari DE and Leon MB: A qualitative and quantitative angiographic analysis of stent fracture late following sirolimus-eluting stent implantation. Am J Cardiol 103: 923-929, 2009.

25. Ranke C, Creutzig A and Alexander K: Duplex scanning of the peripheral arteries: Correlation of the peak velocity ratio with angiographic diameter reduction. Ultrasound Med Biol 18: 433-440, 1992.

26. Shammas NW, Shammas GA, Helou TJ, Voelliger CM, Mrad L and Jerin M: Safety and 1-year revascularization outcome of SilverHawk atherectomy in treating in-stent restenosis of femoropopliteal arteries: A retrospective review from a single center. Cardiovasc Revasc Med 13: 224-227, 2012.

27. Dippel EJ, Makam P, Kovach R, George JC, Patlola R, Metzger DC, Mena-Hurtado C, Beasley R, Soukas P, Colon-Hernandez PJ, et al: Randomized controlled study of excimer laser atherectomy for treatment of femoropopliteal in-stent restenosis: Initial results from the EXCITE ISR trial (EXCImer Laser Randomized Controlled Study for Treatment of FemoropopliTEal In-Stent Restenosis). JACC Cardiovasc Interv 8: 92-101, 2015.

28. Gonzalez L, Chen A, Lin PH, Pisimisis G, Barshes NR Bechara CF and Kougias P: Latest recanalization techniques for complex superficial femoral artery occlusions. J Cardiovasc Surg (Torino) 53: 487-494, 2012.

29. Golomb BA, Dang TT and Criqui MH: Peripheral arterial disease: Morbidity and mortality implications. Circulation 114: 688-699, 2006.

30. He Y, Jiang Y, Wang J, Fan L, Li X and Hu FB: Prevalence of peripheral arterial disease and its association with smoking in a population-based study in Beijing, China. J Vasc Surg 44: 333-338, 2006

31. Luo Y, Li X, Li J, Wang X, Qiao Y, Hu D, Merriam PA and Ma Y: Combined effects of smoking and peripheral arterial disease on all-cause and cardiovascular disease mortality in a Chinese male cohort. J Vasc Surg 51: 673-678, 2010.

32. Dormandy JA and Rutherford RB: Management of peripheral arterial disease (PAD). TASC working group. TransAtlantic inter-society consensus (TASC). J Vasc Surg 31: S1-S296, 2000.

33. Bosiers M, Torsello G, Gissler HM, Ruef J, Müller-Hülsbeck S, Jahnke T, Peeters P, Daenens K, Lammer J, Schroë H, et al: Nitinol stent implantation in long superficial femoral artery lesions: 12-month results of the DURABILITY I study. J Endovasc Ther 16: 261-269, 2009.

34. Zhou W, Bush RL, Lin PH, Peden EK and Lumsden AB: Laser atherectomy for lower extremity revascularization: An adjunctive endovascular treatment option. Vasc Endovascular Surg 40: 268-274, 2006

35. Sultan S, Tawfick W and Hynes N: Cool excimer laser-assisted angioplasty (CELA) and tibial balloon angioplasty (TBA) in management of infragenicular arterial occlusion in critical lower limb ischemia (CLI). Vasc Endovascular Surg 47: 179-191, 2013. 
36. Dave RM, Patlola R, Kollmeyer K, Bunch F, Weinstock BS, Dippel E, Jaff MR, Popma J and Weissman N; CELLO Investigators: Excimer laser recanalization of femoropopliteal lesions and 1-year patency: Results of the CELLO registry. J Endovasc Ther 16: 665-675, 2009.

37. Liistro F, Grotti S, Porto I, Angioli P, Ricci L, Ducci K, Falsini G, Ventoruzzo G, Turini F, Bellandi G and Bolognese L: Drug-eluting balloon in peripheral intervention for the superficial femoral artery: The DEBATE-SFA randomized trial (drug eluting balloon in peripheral intervention for the superficial femoral artery). JACC Cardiovasc Interv 6: 1295-1302, 2013.

38. Rosenfield K, Jafr MR, White CJ, Rocha-Singh K, Mena-Hurtado C, Metzger DC, Brodmann M, Pilger E, Zeller T, Krishnan P, et al: Trial of a paclitaxel-coated balloon for femoropopliteal artery disease. N Engl J Med 373: 145-153, 2015.

39. Tepe G, Laird J, Schneider P, Brodmann M, Krishnan P, Micari A Metzger C, Scheinert D, Zeller T, Cohen DJ, et al: Drug-coated balloon versus standard percutaneous transluminal angioplasty for the treatment of superficial femoral and popliteal peripheral artery disease: 12-month results from the IN.PACT SFA randomized trial. Circulation 131: 495-502, 2015.

40. McKinsey JF, Goldstein L, Khan HU, Graham A, Rezeyat C, Morrissey NJ, Sambol E and Kent KC: Novel treatment of patients with lower extremity ischemia: Use of percutaneous atherectomy in 579 lesions. Ann Surg 248: 519-528, 2008.
41. Wissgott C, Kamusella P, Lüdtke C and Andresen R: Excimer laser atherectomy after unsuccessful angioplasty of TASC C and D lesions in femoropopliteal arteries. J Cardiovasc Surg (Torino) 54: 359-365, 2013.

42. Lüdtke CW, Scheer F, Kamusella P, Wissgott C and Andresen R: Transpopliteal balloon-assisted excimer-laser atherectomy for the treatment of chronic femoropopliteal occlusions: Feasibility and initial results. Clin Med Insights Cardiol 8 (Suppl 2): S23-S28, 2014

43. Sangiorgi G, Lauria G, Airoldi F, Godino C, Politi L, Colombo A, Clerici G, Modena MG and Biondi-Zoccai G: Retrograde popliteal access as bail-out strategy for challenging occlusions of the superficial femoral artery: A multicenter registry. Catheter Cardiovasc Interv 79: 1188-1193, 2012.

(i) (3) This work is licensed under a Creative Commons Attribution-NonCommercial-NoDerivatives 4.0 International (CC BY-NC-ND 4.0) License. 